\title{
Combining effective media and multi-phase methods of Lattice Boltzmann modelling for the characterisation of liquid-vapour dynamics in multi-length scale heterogeneous structural materials
}

P.J. McDonald and M.N. Turner

October 6, 2015

Department of Physics, University of Surrey, Guildford, Surrey, GU2 7XH, UK

\begin{abstract}
The combination of the lattice Boltzmann Shan-Chen pseudo-potential method for multiphase fluids (Shan \& Chen, Phys. Rev. E 47, 1993, 1815) and a grey or partial bounce back lattice Boltzmann algorithm for effective media (Walsh et al, Comp. \& Geosci. 35, 2009, 1186), is demonstrated for application to liquid-vapour fluid dynamics in porous media with porosity spanning a very wide range of length scales. Liquid / vapour distributions in cellular like structures with cell walls of reduced permeability are seen to follow expectation.
\end{abstract}




\section{Introduction}

The lattice Boltzmann method is recognised as a powerful tool for solving equations of fluid dynamics in porous media [1],[2]. The primary advantage is that complex boundaries can be readily incorporated. Several broad classes of method for simulating multiphase and / or multicomponent fluids are well known. These include the "colouring" method proposed by Rothman and Keller [3] and by Gunstensen et al. [4]; the "pseudopotential" method proposed by Shan and Chen, [5],[6]; the "free energy" method proposed by Swift et al. [7] and the mean field method of He et al. [8]. Each of these has been widely developed and augmented by many authors. Overall, the Shan-Chen method has been most widely adopted. Most applications concern multi-component fluid mixtures, such as oil and water. However, it is equally applicable to a single component, multiphase fluid with coexistence of liquid and vapour and with evaporation and condensation between the two as the pressure is varied. In the case of the single component, multiphase fluid, Shan-Chen represents a good compromise between computational efficiency and reality. On the one hand, code that implements it can be made parallel very easily. On the other, the underlying model is not as well grounded in thermodynamics as, say, the free energy method.

A key limitation of all these methods is the computational cost, in terms of memory size and processor time, of adequately simulating fluid dynamics in a porous matrix encompassing a very wide range of structural feature sizes. As one way to overcome this limitation different authors have introduced effective media approaches for single component fluids. Individual lattice nodes on one length scale are assigned generic properties of the underlying material at that node without explicitly describing the underlying material structure at the shorter length scale. Amongst such methods, the partial bounce back, effective media, algorithm proposed by Dardis and McCloskey, [9], and refined by Walsh, Burwinkle and Saar, (WBS), [10], is particularly easy to implement 
and computationally efficient. It has the advantage that, unlike some other schemes, it conserves mass and an analytic expression exists relating the permeability to the effective media parameter.

To the best of our knowledge, no authors have yet attempted to combine multiphase and effective media approaches with the ambition of exploring the transport of a multi-phase fluid in a complex geometry with features at distinctly different, multiple, length scales. We are prompted to do this in order to explore water dynamics in two construction material systems that hitherto have received relatively little attention from the Lattice Boltzmann community: cement and wood. Understanding water dynamics in cement is important because water transport underpins almost all forms of degradation of concrete of which cement is the binder phase. Hydrated cement is a highly heterogeneous material comprising disordered calcium-silicate-hydrates (C-S-H), nano-crystalline solids such as calcium hydroxide, unreacted cement powder and pores. The pores are on a wide range of length scales ranging from nanometre gel pores through to near millimetre sized capillary pores. The largest pores do not generally form a connected network in their own right, but rather are linked by the much finer porosity. Under normal conditions of ambient relative humidity, the smallest pores are water saturated whereas the largest are filled with vapour (and air). A single early lattice Boltzmann study of cement and water, [11], used the single-phase fluid approach to study a model cement structure that only included the capillary pores. More recently, Zalzale and co-workers used the "freeenergy" method to investigate two-phase water sorption in a similar model structure [12] and single-phase effective media methods to explore the effective permeability as a function of degree of water saturation by setting vapour spaces to "solid", again in a model structure [13]. Wood is another porous material used in construction. Again it is heterogeneous on multiple length scales: from the micrometre scale of individual lumen cells and their walls through to the macroscopic structure of tree-rings. Water transport in wood is important both from the perspective of efficient management of living trees 
through to the controlled drying of timber in processing plants. Again there is little prior art in lattice Boltzmann applied to wood. What there is, includes a multiphase analysis of flow using the Shan-Chen method by Frank et al. [14]

Thus the purpose of this paper is to introduce the basic concepts required to combine the Shan-Chen multiphase lattice Boltzmann method with the WBS partial bounce back algorithm in order to address the problem of multiphase fluid dynamics in highly heterogeneous porous media. Preliminary results more immediately relevant to water sorption in wood cells are presented. In a separate communication, we report a parallel study that combines the "free energy" multiphase method with the same partial bounce back algorithm and show preliminary results more appropriate to cement. Further future papers in more specialist journals will explore the materials applications in greater detail.

\section{Theory}

The standard lattice Boltzmann methodology is presented in many texts, including that by Huang et al. [15] that focuses in particular on multiphase methods. The method is encapsulated in the following equation:

$$
f_{i}\left(\mathbf{r}+\mathbf{e}_{i} \Delta t, t+\Delta t\right)-f_{i}(\mathbf{r}, t)=\Delta t \sum_{j=1}^{Q} S_{i, j}\left(f_{i}(\mathbf{r}, t)-f_{i}^{e q}(\mathbf{r}, t)\right)
$$

Here, $f$ is the density of fluid packets travelling with each of $i=1 . . Q$ velocities $\mathbf{e}_{i}$ on a lattice at time $t$ and at position $\mathbf{r}$ such that $\mathbf{e}_{i} \Delta t$ is a lattice vector. The equilibrium density, $f^{e q}$, encompasses the physics of the system under study. The matrix $S$ is the collision matrix that relaxes $f$ towards $f^{e q}$. In single relaxation time simulations it is simply given by

$$
S_{i, j}=-\frac{1}{\tau} \delta_{i, j}
$$


where $\tau$ is a relaxation time related to the kinematic viscosity of the fluid through

$$
\nu=c_{s}^{2}\left(\tau-\frac{\Delta t}{2}\right)
$$

and where

$$
c_{s}=\frac{\Delta x}{\sqrt{3} \Delta t}
$$

is the lattice speed of sound.

For a single phase fluid the equilibrium fluid density is given by

$$
f_{i}^{e q}=\omega_{i} \rho\left(1+\frac{\mathbf{u}^{*} \cdot \mathbf{e}_{i}}{c_{s}^{2}}+\frac{\left(\mathbf{u}^{*} \cdot \mathbf{e}_{i}\right)^{2}}{2 c_{s}^{4}}-\frac{\mathbf{u}^{*} \cdot \mathbf{u}^{*}}{2 c_{s}^{2}}\right)
$$

where the $\omega_{i}$ are lattice choice dependent weight factors, $\rho$ is the fluid density and $\mathbf{u}^{*}$ is the equilibrium velocity that is equal to the macroscopic velocity of the fluid $\mathbf{u}$ in the absence of additional forces. Specifically,

$$
\rho(\mathbf{r}, t)=\sum_{i=1}^{Q} f_{i}(\mathbf{r}, t)
$$

and

$$
\mathbf{u}(\mathbf{r}, \mathbf{t})=\frac{1}{\rho(\mathbf{r}, t)} \sum_{i=1}^{Q} f_{i}(\mathbf{r}, t) \mathbf{e}_{i}
$$

The simulation is run as a series of propagation and relaxation steps. No-slip solid boundaries are effected by bounce back so that for propagation at a solid boundary

$$
f_{i}\left(\mathbf{r}+\mathbf{e}_{i} \Delta t, t+\Delta t\right)=f_{\tilde{i}}(\mathbf{r}, t)
$$

where the tilde implies the opposite direction such that $\mathbf{e}_{i}=-\mathbf{e}_{\tilde{i}}$. 


\subsection{The Shan-Chen multi-phase lattice Boltzmann method}

The Shan-Chen method incorporates multiphase fluids by introducing a fluid potential $\Psi$ dependent on fluid density and thereby a fluid-fluid force term $\mathbf{F}$ dependent on the gradient of the potential that causes phase separation of the fluid into liquid and gas. According to the Shan-Chen implementation the forcing term is given by $[6,16]$

$$
\mathbf{F}=G \Psi(\mathbf{r}) \sum_{i=1}^{Q} \omega_{i}^{a} \mathbf{e}_{i} \Psi\left(\mathbf{r}+\mathbf{e}_{i} \Delta t\right)
$$

where $G$ is the interaction strength constant and $\omega_{i}^{a}$ are force weight coefficients. The potential is designed to lead to pressure-volume characteristics of the fluid that emulate a e.g. van der Waals equation of state. A well known and appropriate function $\Psi$ is

$$
\Psi(\mathbf{r})=\psi_{0} \exp \left(\frac{-\rho_{0}}{\rho(\mathbf{r})}\right)
$$

where $\psi_{0}$ and $\rho_{0}$ are constants. The force can be introduced to the model by modification of the equilibrium velocity, equation [17] so that

$$
\mathbf{u}^{*}=\mathbf{u}+\frac{\tau \mathbf{F}}{\rho}
$$

The associated fluid pressure is given by

$$
P=c_{s}^{2} \rho+12 c_{s}^{2} G \Psi^{2}
$$

Interaction with a solid interface is achieved by assigning a fictitious fluid density, $\rho_{\text {sol }}$ to the solid nodes and hence a constant potential

$$
\Psi(\text { solid })=\psi_{\text {sol }} \exp \left(\frac{-\rho_{0}}{\rho_{\text {sol }}}\right)
$$


The principle limitations of the simple functional form of $\Psi$ are that the gas is more compressible than the liquid and that a large density difference cannot be obtained between gas and liquid. Nonetheless it serves to illustrate the basic concepts.

\subsection{Partial bounceback lattice Boltzmann method}

The WBS partial bounce back algorithm [10] that simulates partially permeable media assigns a variable $\sigma$ ranging from 0 to 1 at every node of the simulation cell. A value of 0 implies that the medium is fully permeable whereas 1 implies solid. The normal rules of propagation and bounce back apply. Partially permeable nodes are assigned a value between 0 and 1 . It is for this reason that partial bounce back methods are often referred to as grey LB and these nodes as grey nodes. Here there is partial bounce back so that a fraction of the fluid $1-\sigma$ moves on as if the node is fluid whereas the remaining fraction, $\sigma$ bounces back as if the node were solid. ${ }^{1}$ The relaxation and propagation step is therefore expressed everywhere as:

$$
f_{i}\left(\mathbf{r}+\mathbf{e}_{i} \Delta t, t+\Delta t\right)=(1-\sigma)\left[\left(1-\frac{\delta t}{\tau}\right) f_{i}(\mathbf{r}, t)+\frac{\delta t}{\tau} f_{i}^{e q}(\mathbf{r}, t)\right]+\sigma f_{\tilde{i}}(\mathbf{r}, t)
$$

The parameter $\sigma$ is related to the effective permeability through ${ }^{2}$

$$
\kappa=\frac{1-\sigma}{2 \sigma} \nu \Delta t
$$

\footnotetext{
${ }^{1} \mathrm{Zhu}$ and $\mathrm{Ma},[18]$, take pains to point out that $\sigma$ is a model parameter but is not, in this particular implementation, strictly a solids fraction since the density function propagated, $f^{e q}$, and bounced back, $f$, are different. They offer an improved, but computationally more complex, model where this is not the case: the same function is propagated and bounced back.

${ }^{2}$ In literature, Eq. 15 appears without the factor $\Delta t$. Dimensionally, it is clearly required and it follows from a careful analysis of Darcy's law (Equation 11 in reference [10]) paying strict attention to the use of physical and simulation space and time steps. In the event that $\Delta t=1$, its omission is of no numerical consequence
} 


\subsection{The combined method}

In order to combine the Shan-Chen multiphase and WBS effective media frameworks, we everywhere write the potential as

$$
\Psi_{c m}(\mathbf{r})=(1-\sigma(\mathbf{r})) \psi_{0} \exp \left(\frac{-\rho_{0}}{\rho(\mathbf{r})}\right)+\sigma(\mathbf{r}) \psi_{s o l} \exp \left(\frac{-\rho_{0}}{\rho_{s o l}}\right)
$$

the subscript $\mathrm{cm}$ standing for combined method. The force term, equilibrium velocity and pressure follow directly from equations 9 to 12 :

$$
\begin{gathered}
\mathbf{F}_{c m}=G \Psi_{c m}(\mathbf{r}) \sum_{i=1}^{Q} \omega_{i}^{a} \mathbf{e}_{i} \Psi_{c m}\left(\mathbf{r}+\mathbf{e}_{i} \Delta t\right) \\
\mathbf{u}^{*}=\mathbf{u}+\frac{\tau \mathbf{F}_{c m}}{\rho}
\end{gathered}
$$

and

$$
P_{c m}=c_{s}^{2} \rho+12 c_{s}^{2} G \Psi_{c m}^{2}
$$

respectively. The factor $12 c_{s}^{2}$ in the second term of equation 19 which equals 4 in the case $\Delta x=\Delta t=1$ was verified experimentally and agrees with Sukop and Or in the case $\sigma=0$. Finally, equation 14 is used for propagation and relaxation with the equilibrium function calculated with equations 5 and 18 .

\section{Methods}

Simulations can be run on a variety of lattices but D2Q9 (2-dimensions, 9 velocity directions) and D3Q27 are popular choices. In this work, we chose D2Q9 for which case 
the velocity vectors, lattice and force weights are:

$$
\begin{aligned}
\mathbf{e} & =([0,0],[0,1],[\overline{1}, 0],[0, \overline{1}],[1,0],[1,1],[1, \overline{1}],[\overline{1}, \overline{1}],[\overline{1}, 1]) \\
\omega & =(4 / 9,1 / 9,1 / 9,1 / 9,1 / 9,1 / 36,1 / 36,1 / 36,1 / 36) \\
\omega^{a} & =(0,2,2,2,2,1,1,1,1)
\end{aligned}
$$

respectively

The parameters $G, \psi_{0}, \rho_{0}, \psi_{\text {sol }}, \rho_{\text {sol }}$ and $\sigma$ offer considerable flexibility in defining the fluid interaction with itself, with solid walls and with semi-permeable media. Indeed, the "solid" parameters can be made functions of $\sigma$. In this work we chose however to simplify matters considerably by allowing just three values of $\sigma$ : 0 representing free fluid; 1 representing solid; and a single intermediate value in any one simulation. We also set $\psi_{0}=\psi_{\text {sol }}$. These simplifications are made at the expense of opening a far wider parameter space and being able to fine-tune the system to best match a given physical system. In doing so, we use fluid-fluid interaction potential parameter values proposed by Sukop and Or: $G=-5$ and $\psi_{0}=4$ and $\rho_{0}=200 \mathrm{mu} \cdot \mathrm{lu}^{-2}$.

For the most part, periodic boundary conditions were adopted in both directions. However, for results presented towards the end of this report, pressure boundary conditions using the Zou and He [19] pressure boundary conditions were implemented along boundary regions of free fluid.

A code to implement the combined algorithm and run simulations was written in Matlab with $\delta x=\delta t=\tau=1$. This considerably simplifies equation to 14

$$
f_{i}\left(\mathbf{r}+\mathbf{e}_{i} \Delta t, t+\Delta t\right)=(1-\sigma) f_{i}^{e q}(\mathbf{r}, t)+\sigma f_{\tilde{i}}(\mathbf{r}, t)
$$

It is noted that for the study of sorption and transport in porous media, the flow is creeping and so the choice of viscosity, defined by the choice $\tau=1$, is not expected 
to affect the equilibrium results. The code was validated against previously published results. Simulations were judged to have converged when the root mean square fractional change in density per cycle per lattice node was less than $10^{-8}$ or better. By setting $G=$ 0 , the multiphase interaction is turned off and the code reduces to an implementation of the WBS model. Results published by Walsh et al. were reproduced. In particular the permeability of flow channels of uniform $\sigma$ was measured and equation 15 was verified over the full range of $\sigma$. By setting $G=-5$ and $\sigma=0$ everywhere the code reduces to an implementation of the Shan-Chen model. The expected phase separation was seen. The pressure across flat interfaces was seen to be zero and the Kelvin Laplace equation was used to check that the pressure varied with radius of droplets as expected. In all cases, literature results were readily reproduced.

\section{Results}

\subsection{Equation of state and coexistence densities}

Figure 1 is a plot of the effective equation of state, Eq. 19, for different sets of parameters. It is a plot of pressure against reciprocal density, so making the figure appear like a regular pressure-volume plot. The solid curve is for $\sigma=0$ using the Shan-Chen parameters investigated by Sukop and Or. The standard equal-area Maxwell construction yields coexistence gas and liquid densities of 78 and $512 \mathrm{mu} \cdot \mathrm{lu}^{-2}$. These are indicated by the ends of the thin horizontal line drawn on the plot. The other lines in the Figure show how the Equation of State changes for selected values of $\sigma$ and $\rho_{\text {sol }}$. As $\sigma$ is increased while keeping $\rho_{\text {sol }}=0$ so the curve between the coexistence densities tends to flatten until such time as the curve decreases monotonically from high to low pressure and no phase separation can occur. The dashed curve is plotted for $\sigma=0.1$, just short of the critical value for no phase separation when $\rho_{\text {sol }}=0$. In this case the coexistence densi- 


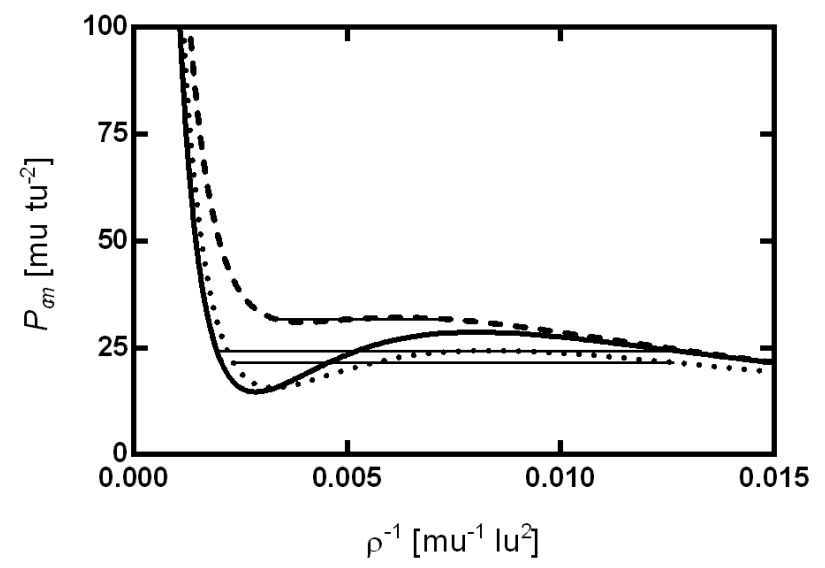

Figure 1: Effective equation of state for the Shan-Chen model using $G=-5, \psi_{0}=4$ and $\rho_{0}=200 \mathrm{mu} . \mathrm{lu}^{-2}$ with $\sigma=0$ (solid line) ; with $\sigma=0.1$ and $\rho_{\text {sol }}=0 \mathrm{mu} \cdot \mathrm{lu}^{-2}$ (dashed line); and with $\sigma=0.1$ and $\rho_{\text {sol }}=300 \mathrm{mu} \cdot \mathrm{lu}^{-2}$ (dotted line). The thin solid horizontal lines connect the co-existence densities for each curve as in the equal-area Maxwell construction.

ties are 135 and 298 mu.lu ${ }^{-2}$. On the other hand, as $\rho_{\text {sol }}$ is increased, so the minimum and maximum in the curve are enhanced, strengthening the fluid phase separation in the partially permeable medium. A curve is drawn for $\sigma=0.1$ and $\rho_{\text {sol }}=300 \mathrm{mu} \cdot \mathrm{lu}^{-2}$, dotted line. It is similar to the original curve. The coexistence densities are 79 and 428 mu.lu ${ }^{-2}$.

The coexistence densities can be measured by observing phases separation in a lattice Boltzmann simulation. For a small rectangular simulation cell of 4 by 100 nodes initialised with a fluid of uniform intermediate density with small random fluctuations to seed phase separation, the fluid phase separates into liquid and gas parts with a straight interface. For the case of $\sigma=0$, and using Sukp ond Or's parameters, the densities are 85 and $524 \mathrm{mu} \cdot \mathrm{lu}^{-2}$. These values are close to the expected values from the equal area Maxwell construction and in agreement with the gas density used by Sukop and Or (2003). The experimental dependence of the gas and liquid coexistence densities has been systematically measured in a similar way for different values of the variable param- 


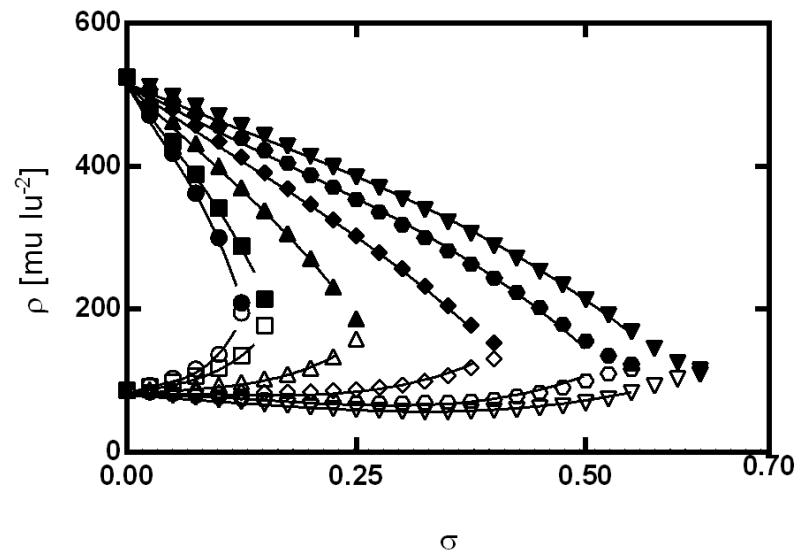

Figure 2: Coexistence densities for liquid (solid symbols) and vapour (open symbols) as a function of $\sigma$ for $\rho_{\text {sol }}=0$ (circles), 100 (squares), 200 (triangles), 300 (diamonds), 400 (hexagons) and $500 \mathrm{mu} \cdot \mathrm{lu}^{-2}$ (inverted triangles) determined from the results of lattice Boltzmann simulations in a rectangular simulation cell of 4 by 100 nodes. In each case, at values of $\sigma$ above that at which the two curves meet, only one fluid phase is observed. The solid lines show the same densities found by numerically solving the equal-area Maxwell construction for the equation of state, Eq. 19

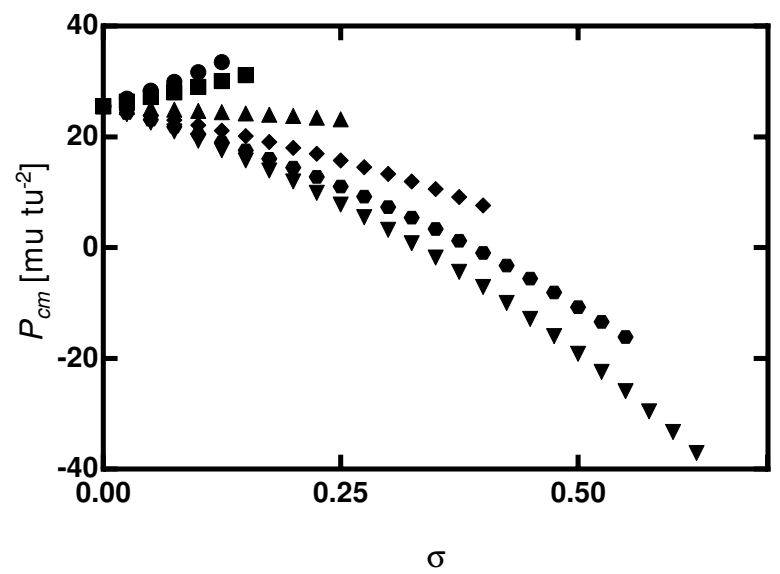

Figure 3: The vapour/liquid coexistence pressure as a function of $\sigma$ for $\rho_{\text {sol }}=0$ (circles), 100 (squares), 200 (triangles), 300 (diamonds), 400 (hexagons) and $500 \mathrm{mu} . \mathrm{lu}^{-2}$ (inverted triangles). 
eters $\sigma$ and $\rho_{\text {sol }}$. In each simulation, $\sigma$ was made uniform across the cell and the system initiated to a uniform intermediate density. The results are plotted as a function of $\sigma$ for different $\rho_{\text {sol }}$ in Figure 2. The plots make clear how, for any given $\rho_{\text {sol }}$, there is a critical $\sigma$ above which phase separation does not occur and that this critical value increases with $\rho_{\text {sol }}$. The figure also includes solid lines which are curves through points found by numerical solution of the equal area Maxwell construction for the equation of state. There is evidently good agreement between the two. Figure 3 shows the corresponding pressure calculated from the Lattice Boltzmann simulations for the same parameter sets. The results give us confidence in Equation 19.

\subsection{Homogeneous media}

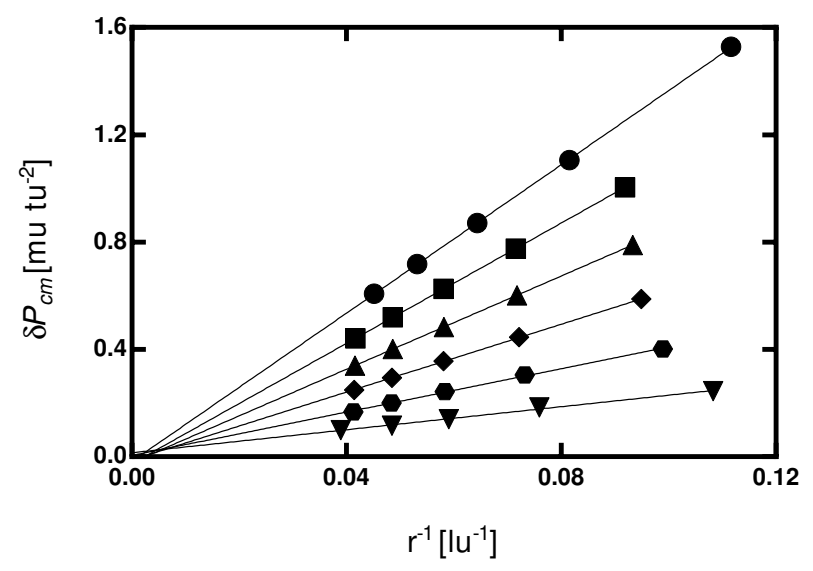

Figure 4: Pressure difference across a drop interface as a function of reciprocal drop radius for $\rho_{\text {sol }}=300 \mathrm{mu} . \mathrm{lu}^{-2}$ and different $\sigma$ : 0 , circles; 0.05, squares; 0.1, triangle, 0.15: dimaonds; 0.2 , hexagons and 0.25 inverted triangles. The straight lines are least square fits to the data.

Liquid in vapour drops, and vice versa, in semi-permeable media can be readily simulated by constructing simulation cells of low aspect ratio (e.g. square) in which every node has the same constant value of $\sigma$. Figure 4 shows a plot of the pressure difference inside versus outside a drop for $\rho_{\text {sol }}=300 \mathrm{mu} \cdot \mathrm{lu}^{-2}$ as a function of the reciprocal drop radius 


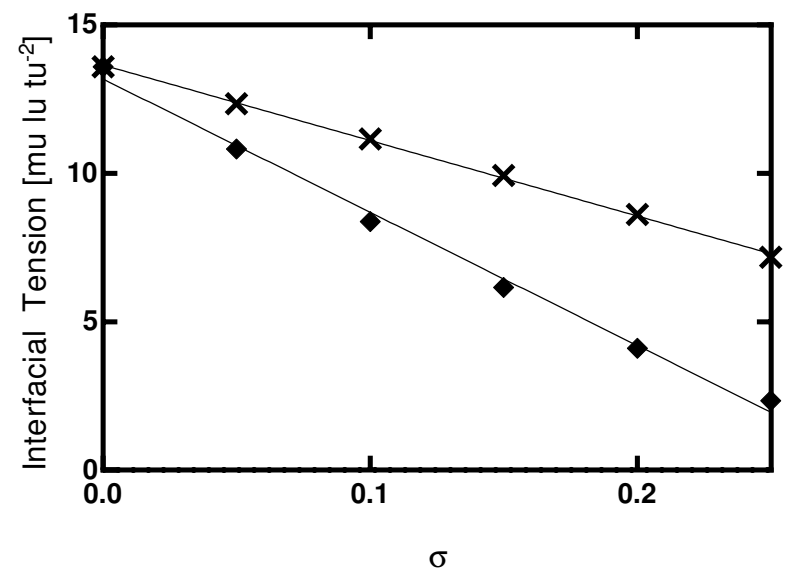

Figure 5: The interfacial tension calculated as the gradient of pressure difference against reciprocal radius $(\partial \delta P / \partial(1 / r))$ as a function of $\sigma$ for $\rho_{\text {sol }}=300$ (diamonds) and 500 mu.lu ${ }^{-2}$ (crosses). The lines are least square fits to the data.

for different $\sigma$. In each case the data follow a straight line that passes through the origin in accordance with the Kelvin Laplace equation. The gradient of the fit line decreases markedly with increasing $\sigma$ showing that the interfacial tension is reducing. This is physically realistic as the contribution of the fluid-fluid interaction to the combined potential (at constant $\rho_{\text {sol }}$ ) decreases as $\sigma$ is increased. The gradient corresponds to the interfacial tension. Figure 5 shows this as a function of $\sigma$ for two different values of $\rho_{\text {sol }}$. It reveals two things: first that the interfacial tension is linear in $\sigma$; and second that for constant $\sigma$ the interfacial tension is stronger for a larger value of $\rho_{\text {sol }}$ which makes sense because increasing $\rho_{\text {sol }}$ increases the fluid potential, $\Psi_{c m}$.

\subsection{Straight interfaces}

A range of bubbles that straddle a straight interface between a region of effective media $(\sigma>0)$ and free space $(\sigma=0)$ can be observed for different values of the parameters and fluid content. As an example, the first panel of Figure 6 shows a structure of $80 \times 80$ nodes 

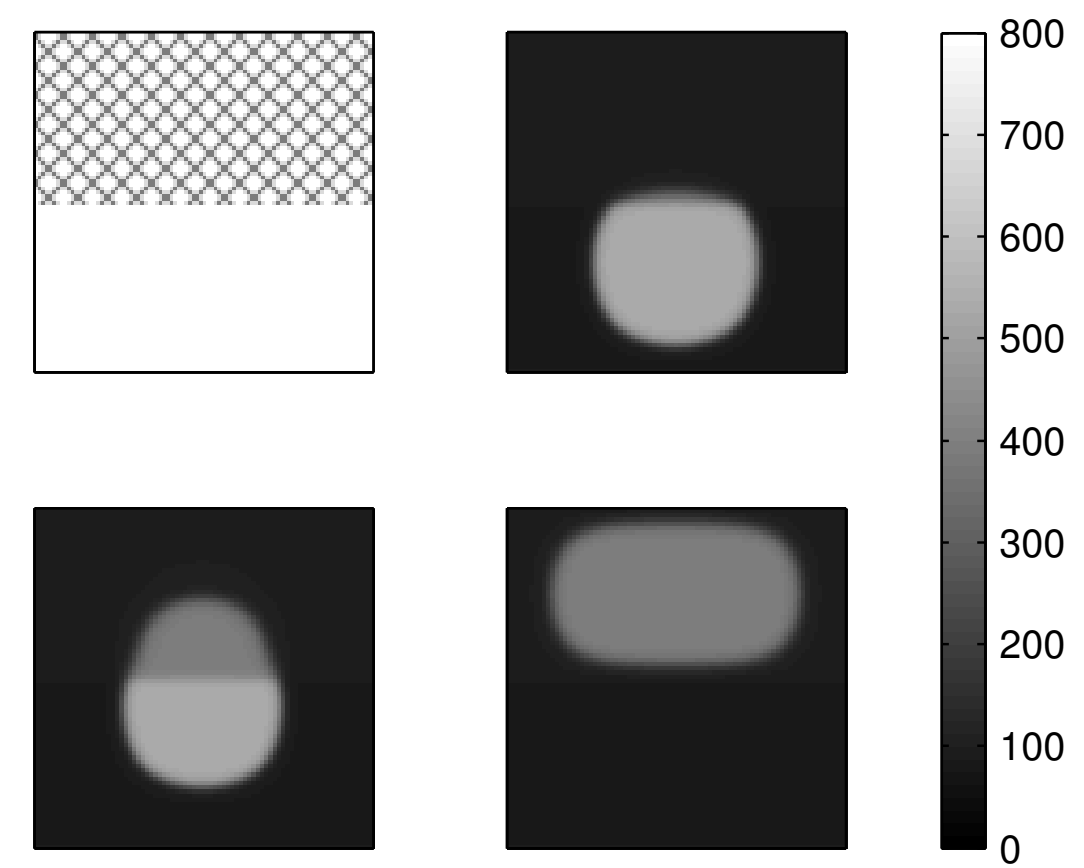

$-300$

200

100

Figure 6: The top left panel shows a region for which the upper half has $\sigma=0.1$, the lower half $\sigma=0$. The remaining panels show a liquid drop equilibrated in this region for different values of $\rho_{\text {sol }}$ : 164 (top right), 172 (lower left) and $180 \mathrm{mu}^{-l u^{-2}}$ (lower right) . The colour bar ranges from density 0 to $800 \mathrm{mu} \cdot \mathrm{lu}^{-2}$ 


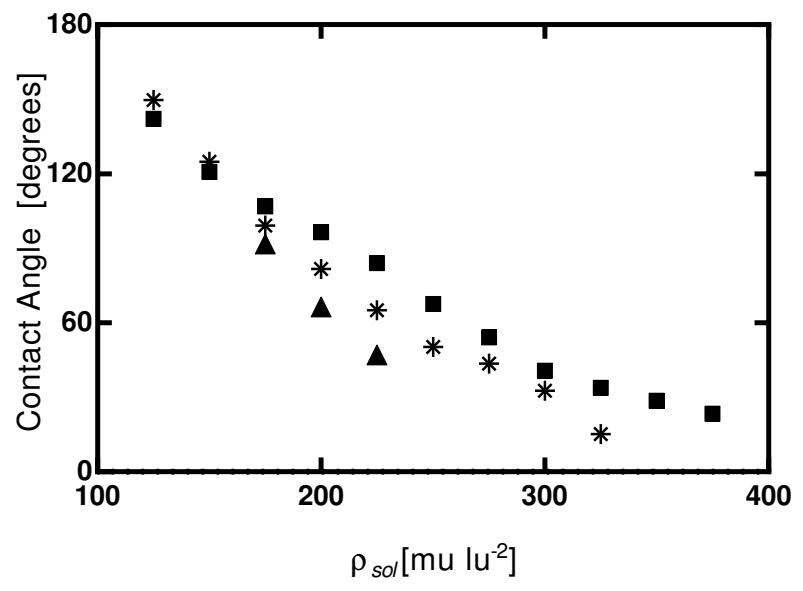

Figure 7: The contact angle formed by a liquid bubble contacting a planar solid interface as a function of $\rho_{\text {sol }}$ for $\sigma=0$ (squares), 0.1 (stars) and 0.2 (triangles)

of which the upper half has $\sigma=0.1$ and the lower half $\sigma=0$. The remaining panels show liquid bubbles equilibrated in this structure. In each case, the cell was initiated to a density $\rho=78 \mathrm{mu} \cdot \mathrm{lu}^{-2}$ (gas) everywhere except for a central circle of radius 20 lu where it was initiated to $512 \mathrm{mu} \cdot \mathrm{lu}^{-2}$ (liquid). They differ in the parameter $\rho_{\text {sol }}$. In the first case, $\rho_{\text {sol }}=164 \mathrm{mu} \cdot \mathrm{lu}^{-2}$ and the bubble is seen to have equilibrated in the lower region. The liquid has moved to the region with with $\sigma=0$ indicating that the semi-permeable region de-wets. The last panel shows what happens when $\rho_{\text {sol }}=180 \mathrm{mu} . \mathrm{lu}^{-2}$. The liquid moves to the semi-permeable region, indicating that it wets. However, the liquid density is lower than before, consistent with the data in Figure 2. Intermediate behaviour is seen for for an intermediate value, $\rho_{\text {sol }}=172 \mathrm{mu} \cdot \mathrm{lu}^{-2}$ in the remaining panel of the Figure. The liquid density within the drop is greater in the free space region compared to the effective media region.

Figure 7 is a plot of the contact angle made by a vapour bubble in a uniform semipermeable medium of constant $\sigma$ contacting a straight solid surface $(\sigma=1)$ for three values of $\sigma_{\text {sol }}: 0,0.1$ and 0.2 . The contact angle was measured empirically from plots of the 
data. The estimated error in this measurement is $\pm 10^{\circ}$. The data show a relatively weak dependence of the contact angle on $\rho_{\text {sol }}$.

\subsection{Inhomogeneous and porous media}

The primary envisaged application area for this combined methodology is the study of fluid sorption and transport in porous media with porosity on multiple length scales. Large pores and solids can be modelled explicitly $(\sigma=0,1$ respectively) ; regions with finer porosity are treated as semi permeable and assigned an intermediate value of $\sigma$. Examples of interest to the authors include wood lumen cells represented as partially permeable cell walls bounding large cellular spaces or the microstructure of cement paste comprising unreacted cement and calcium hydroxide solids, nanoporous calcium-silicatehydrate gel and large ( $>$ micron) capillary pores.

In the case of wood lumen cells, at full hydration water is found in the cell spaces as well as in the cell walls. As the water content is lowered, for instance by equilibrating at lower relative humidity, so liquid water is lost first from the cell space and then the cell wall to be replaced by vapour. In the case of cement, at full saturation the capillary and gel porosity is full of liquid water. As the water content is lowered so the capillary porosity empties first even though these pores are not directly connected to the surface. This basic behaviour is mirrored by the combined Shan-Chen, WBS algorithm. The first panel of Figure 8 shows the fluid distribution in a test cell comprising a low permeability cell wall $\left(\sigma=0.3, \rho_{\text {sol }}=300\right)$ and cell space $(\sigma=0)$. The matrix is $64 \times 32$ nodes and the elliptical central space has major and minor radii 27 and 12 lu respectively. The remaining panels of the Figure show in sequence the result of equilibrating fluid in the cell structure at progressively lower average density, from $524 \mathrm{mu} \cdot \mathrm{lu}^{-2}$ down in equal steps to $524 / 12 \mathrm{mu} . \mathrm{lu}^{-2}$. The first panel of the series shows high concentration of liquid water (light tones) in the cell space and a lower concentration of liquid (mid tones) in 
the cell wall. At an intermediate average density of $306 \mathrm{mu} . \mathrm{lu}^{-2}$, a vapour bubble (dark tones) is seen in the middle of the cell space with the remaining liquid water confined to the top and bottom more highly curved regions of the space and, at lower density, in the cell walls. By 262 mu.lu ${ }^{-2}$ average density, very little liquid remains in the cell space, what there is now contacting the side walls. At lower average density again, vapour entirely fills the cell space although there is still liquid in the walls. Only at the lowest average density, at the end of the series, is there vapour everywhere.

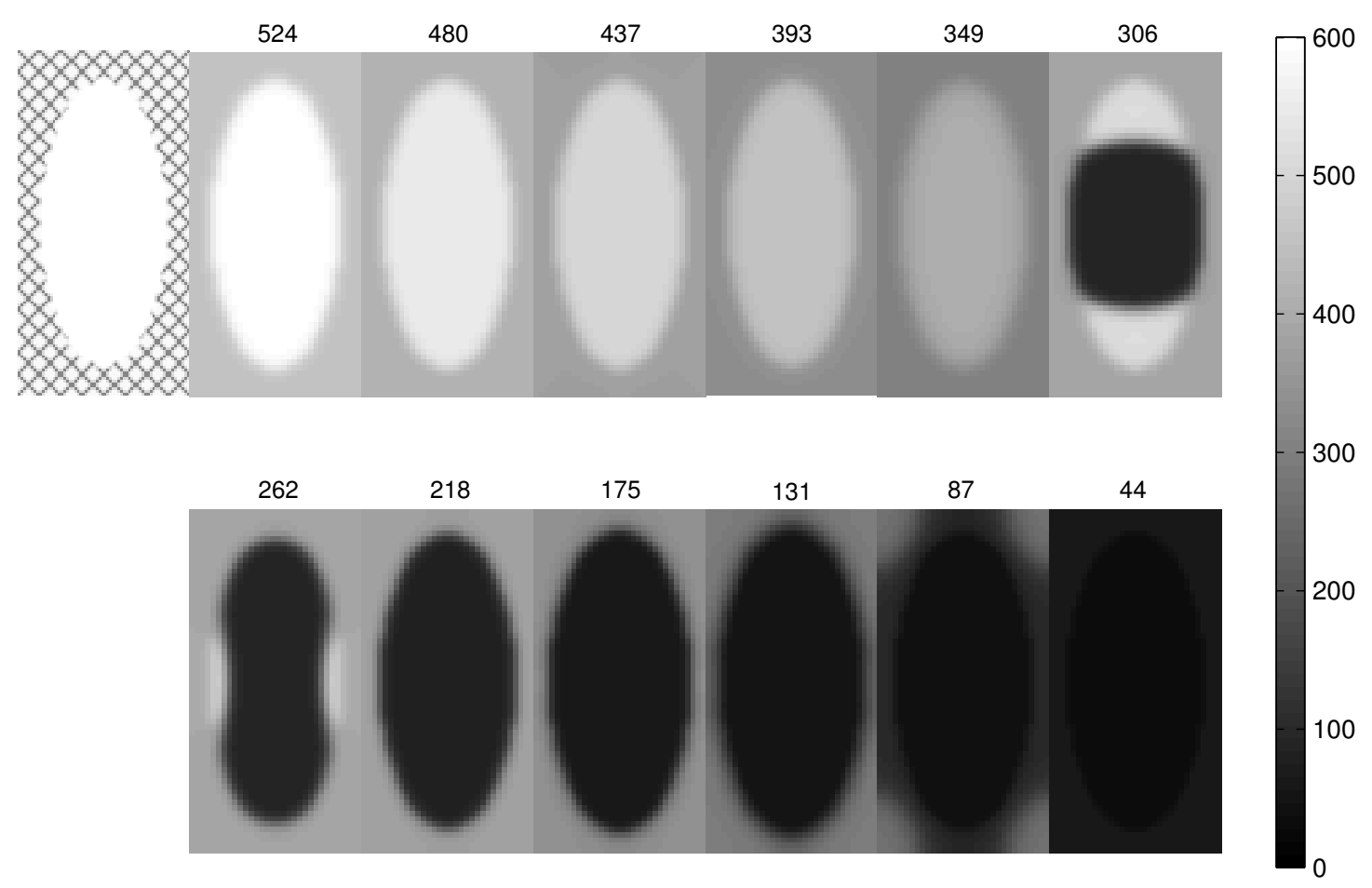

Figure 8: The leftmost plot of the top row is a map of $\sigma$ for a stylised lumen cell comprising a central lumen space $(\sigma=0)$ and a surrounding cell wall $(\sigma=0.3)$. The map for $\rho_{\text {sol }}$ is the visually the same: $\rho_{\text {sol }}=300$ where $\sigma=0.3$. Thereafter, the greyscale maps show the equilibrated distribution of fluid density in the cell and cell wall as the total water content is progressively decreased from left to right and top to bottom in equal steps, from an average density of 524 to $44 \mathrm{mu} . \mathrm{lu}^{-2}$ per node. The greyscale ranges from dark (vapour) to lighter tones (liquid).

Finally, Figure $9 a$ shows the fluid density in a lattice of $15 \times 5$ cell spaces separated by 
semi-permeable walls each one similar to that in Figure 8. A real wood structure would not comprise a regular lattice of cells but using one serves to highlight the differences between spaces and walls. Each cell is elliptical with major and minor radii of 8 and $3.5 \mathrm{lu}$ respectively. The total matrix size is $300 \times 100 \mathrm{lu}^{2}$. The bottom boundary which includes a region of free fluid $(\sigma=0) 3 \mathrm{lu}$ wide is maintained at the bulk liquid coexistence density $\left(524 \mathrm{mu} \cdot \mathrm{lu}^{-2}\right)$ and the top boundary is similarly maintained at $60 \%$ of the coexistence gas density, that is $47 \mathrm{mu} . \mathrm{lu}^{-2}$. The set-up therefore simulates liquid ingress from the bottom and drying from the top. Gravity is not included and periodic boundary conditions apply side to side. The simulation leading to the primary map is carried out for $\rho_{\text {sol }}=300 \mathrm{mu} \cdot \mathrm{lu}^{-2}$ and $\sigma=0.3$. There is a clear drying front two fifths of the way down the structure between cells near the bottom that clearly contain liquid and cells near the top that contain vapour. Connectivity between the cells is maintained by the semi-permeable walls which contain liquid or vapour as might be expected. The other parts of the Figure shows average density profiles down the centre of the system for a set of parameters $\sigma$ and $\rho_{\text {sol }}$ systematically varied around the part $a$ values. Increasing $\rho_{\text {sol }}$ provides stronger fluid density demarcation between the cells and cell walls in both the liquid and vapour occupied regions: compare parts $(c, e)$ to $(b, d)$. The liquid / vapour interface is nearer the drying surface and the density difference averaged over the cell length is sharper for lower values of $\sigma$ : compare parts $(b, c)$ to $(d, e)$. It is therefore evident that these two parameters alone offer significant opportunity to manipulate the drying characteristics of the network. No doubt if further parameters of the model were allowed to vary, then greater control still could be exercised. 
(a)

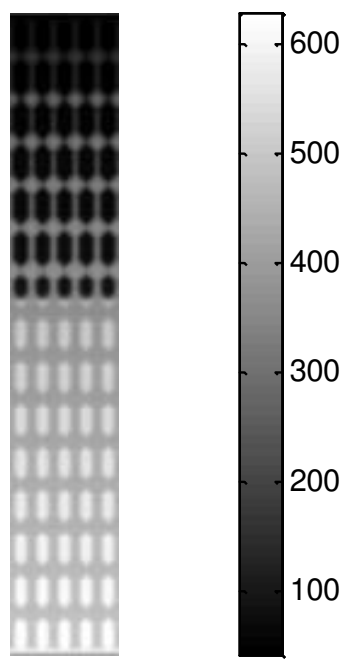

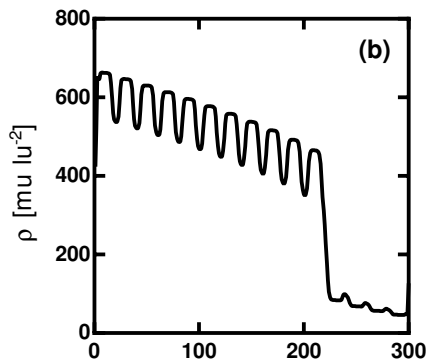
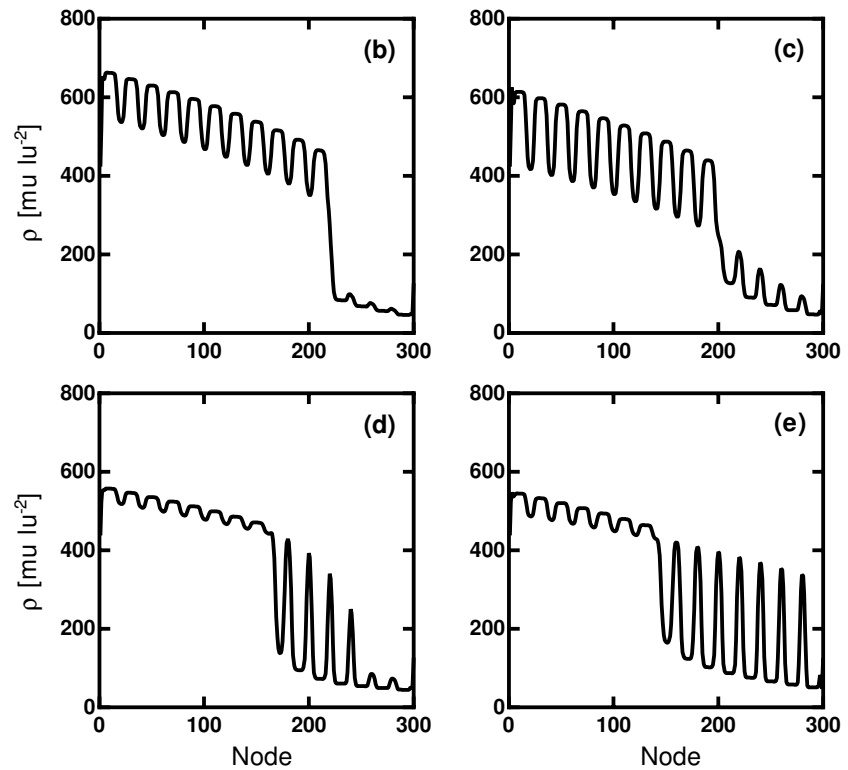

Figure 9: Part $a$ of the figure shows the liquid density in a matrix of cells similar to those in figure 8 with $\rho_{\text {sol }}=300 \mathrm{mu} \cdot \mathrm{lu}^{-2}$ and $\sigma=0.3$. The lower boundary is held at a density of $524 \mathrm{mu} \cdot \mathrm{lu}^{-2}$ representing a wetting condition and the top at $0.6 \times 78=47$ mu.lu $\mathrm{u}^{-2}$ representing a drying condition. There is a clear drying front about $40 \%$ of the way down the sample. The colourbar ranges from dark tones (vapour) to light tones (liquid). The other parts of the Figure show the average density down the centre of the structure for $b:\left(\rho_{\text {sol }}, \sigma\right)=(200,0.2) ; c:(400,0.2) ; d:(200,0.4) ; e:(400,0.4)$; 


\section{Discussion}

\subsection{Alternate multiphase and partial bounceback models}

We chose to implement the WBS variant of partial bounce back on the grounds of computational simplicity but also because it conserves mass in the propagation step. Not all variants of bounce back share this property as shown by Walsh et al and further explored in greater detail by Zhu and Ma, [18]. Walsh et al. observe that momentum is not conserved in general in the partial bounce back method for heterogenous $\sigma$, their variant included. There are spurious mass flows in the region of material interfaces. This is true of the combined model too: the limitations of partial bounce back carry over. We have made a similar analysis that combines the WBS algorithm with the free energy lattice Boltzmann multiphase model of fluids as proposed by [7] and further developed by Yeomans and co-workers [20]. This work is to be published elsewhere but is also described in the thesis work of Zalzale [21]. We find that the basic concepts carry across. In particular, this alternate approach permits easy differentiation of transport and wetting parameters. Such differentiation has not been yet achieved algebraically for the model described here. The principle limitation of the alternate approach is that the computational cost is somewhat greater and overall the simulation is less numerically stable.

\subsection{Outlook}

Beyond the limitations of partial bounce back or grey lattice Boltzmann method just noted, as of today we see two major hurdles that need to be overcome to make the method routinely useful to the materials scientist. The first is that a forcing term that better represents the behaviour of real complex fluids such as water is required. There are two aspects to this. One is the substantially larger density difference between vapour 
and liquid than is achieved in this work. The second is that with the potential used here, the gas is more compressible than the liquid. Others recognise and are addressing these issues, [22] [23] [24] The second is that both wood and cement are dynamic structures. This is relatively obvious in the case of wood: as wood dries so its macroscopic shape and size changes (warping and shrinkage) reflecting structural changes on the microscale. Such changes also occur in cement. Drying shrinkage is a well known phenomenon of engineering importance, just less visually obvious and there is experimental evidence on nanoscopic pore rearrangements. In the future, models that dynamically allow the boundaries of cellular structures to move in response to the fluid pressure will be required. Others are working towards achieving this goal, [25]. Finally, dynamic semi-permeable media will enable cell-wall "pits" to be introduced into studies of drying in wood. Pits are most simply understood as fluid "gates" in cell walls that open or close dependent on the difference in fluid pressure either side.

\section{Conclusion}

In this paper we have shown that a combined multiphase and partial bounce back algorithm offers interesting opportunities for modelling fluid dynamics in heterogeneous materials with heterogeneity spanning a very wide range of typical sizes. Preliminary results show that the sorption of water into model pores or cells with low permeability / microporous walls qualitatively follows the expected pattern of uptake and distribution at different fluid contents. By varying just two parameters, a wide variety of media can be modelled. In particular it is possible to arrange for droplets to condense either in the open pore space or in the low permeability material. Yet further parameters can be varied in future allowing the model to be carefully tailored to different circumstances and systems. 


\section{Acknowledgements}

The authors thank M. Zalzale recently of EPFL, Lausanne, Switzerland for useful discussions.

\section{References}

[1] C. K. Aidun, J. R. Clausen, Lattice-Boltzmann method for complex flows, Annual review of fluid mechanics 42 (2010) 439-472.

[2] H. Liu, Q. Kang, C. R. Leonardi, B. D. Jones, S. Schmieschek, A. Narváez, J. R. Williams, A. J. Valocchi, J. Harting, Multiphase lattice Boltzmann simulations for porous media applications - a review, arXivarXiv:1404.7523v1.

[3] D. H. Rothman, J. M. Keller, Immiscible cellular-automaton fluids, J Stat Phys 52 (1988) 1119-1127.

[4] A. K. Gunstensen, D. H. Rothman, S. Zaleski, G. Zanetti, Lattice Boltzmann model of immiscible fluids, Phys. Rev. A 43 (1991) 4320-4327.

[5] X. Shan, H. Chen, Lattice Boltzmann model for simulating flows with multiple phases and components, Phys. Rev. E 47 (1993) 1815-1819.

[6] X. Shan, H. Chen, Simulation of nonideal gases and liquid-gas phase transitions by the lattice Boltzmann equation, Phys. Rev. E 49 (1994) 2941-2948.

[7] M. R. Swift, E. Orlandini, W. R. Osborn, J. M. Yeomans, Lattice Boltzmann simulations of liquid-gas and binary fluid systems, Phys. Rev. E 54 (5) (1996) 5041.

[8] X. He, S. Chen, R. Zhang, A lattice Boltzmann scheme for incompressible multiphase flow and its application in simulation of Rayleigh-Taylor instability, Journal of Computational Physics 152 (1999) 642-663. 
[9] O. Dardis, J. McCloskey, Lattice Boltzmann scheme with real numbered solid density for the simulation of flow in porous media, Phys. Rev. E 57 (1998) 4834-4837.

[10] S. D. C. Walsh, H. Burwinkle, M. O. Saar, A new partial-bounceback latticeBoltzmann method for fluid flow through heterogeneous media, Computers \& Geosciences 35 (6) (2009) 1186-1193.

[11] E. J. Garboczi, D. P. Bentz, The effect of statistical fluctuation, finite size error, and digital resolution on the phase percolation and transport properties of the NIST cement hydration model, Cem. Conc. Res. 31 (2001) 1501-1514.

[12] M. Zalzale, P. J. McDonald, Lattice Boltzmann simulations of the permeability and capillary adsorption of cement model microstructures, Cem. Conc. Res. 42 (12) (2012) 1601-1610.

[13] M. Zalzale, P. J. McDonald, K. L. Scrivener, A 3D lattice Boltzmann effective media study: understanding the role of C-S-H and water saturation on the permeability of cement paste, Modelling Simul. Mater. Sci. Eng. 21 (8) (2013) 085016.

[14] X. Frank, G. Almeida, P. Perré, Multiphase flow in the vascular system of wood: From microscopic exploration to 3-D Lattice Boltzmann experiments, International Journal of Multiphase Flow 36 (8) (2010) 599-607.

[15] H. Huang, M. C. Sukop, X.-y. Lu, Multiphase Lattice Boltzmann Methods: Theory and Application, Wiley-Blackwell, 2015.

[16] M. C. Sukop, D. Or, Lattice Boltzmann method for modeling liquid-vapor interface configurations in porous media, Water Resources Research 40 (1) (2004) 1-11.

[17] A. A. Mohamad, A. Kuzmin, A critical evaluation of force term in lattice Boltzmann method, natural convection problem, International Journal of Heat and Mass Transfer 53 (2010) 990-996. 
[18] J. Zhu, J. Ma, An improved gray lattice Boltzmann model for simulating fluid flow in multi-scale porous media, Advances in Water Resources 56 (2013) 61-76.

[19] Q. Zou, X. He, On pressure and velocity boundary conditions for the lattice Boltzmann BGK model, Phys. Fluids 9 (6) (1997) 1591-1598.

[20] J. M. Yeomans, Mesoscale simulations: Lattice Boltzmann and particle algorithms, Physica A: Statistical Mechanics and its Applications 369 (1) (2006) 159-184.

[21] M. Zalzale, Water dynamics in cement paste: insights from lattice Boltzmann modelling, Ph.D. thesis, Ecole Polytechnique de Lausanne (Jul. 2014).

[22] T. Lee, C. L. Lin, A stable discretization of the lattice Boltzmann equation for simulation of incompressible two-phase flows at high density ratio, Journal of Computational Physics.

[23] H. W. Zheng, C. Shu, Y. T. Chew, A lattice Boltzmann model for multiphase flows with large density ratio, Journal of Computational Physics 218 (1) (2006) 353-371.

[24] D. Lycett-Brown, K. H. Luo, Improved forcing scheme in pseudopotential lattice Boltzmann methods for multiphase flow at arbitrarily high density ratios, Phys Rev E $91(2015) 1-12$.

[25] X. Chen, Z. Wang, D. Deep, E. Lima, Y. Zhao, S. D. Teague, Mass-conserved volumetric lattice Boltzmann method for complex flows with willfully moving boundaries, Phys. Rev. E 89 (2014) 063304. 